\title{
TSL/OSL/RPL Automated and Integrated Measurement System (TORAIMS)
}

\author{
Go Okada, ${ }^{1 *}$ Kazuki Hirasawa, ${ }^{1}$ Takayuki Yanagida,${ }^{2}$ and Hidehito Nanto ${ }^{1,3}$ \\ ${ }^{1}$ Co-creative Research Center of Industrial Science and Technology (CIST), Kanazawa Institute of Technology, \\ 3-1 Yatsukaho, Hakusan, Ishikawa 924-0838, Japan \\ ${ }^{2}$ Division of Materials Science, Nara Institute of Science and Technology (NAIST), \\ 8916-5 Takayama, Ikoma, Nara 630-0192, Japan \\ ${ }^{3}$ Oarai Research Center, Chiyoda Technol Corporation, \\ 3618 Narita-cho, Oarai-machi, Higashi-ibaraki-gun, Ibaraki 311-1313, Japan
}

(Received January 31, 2021; accepted March 22, 2021; online published March 26, 2021)

Keywords: TSL, OSL, RPL, automation, integrated measurement, radiation measurement, phosphor

Thermally stimulated luminescence (TSL), optically stimulated luminescence (OSL), and radio-photoluminescence (RPL) are radiation-induced luminescence phenomena, and they are widely used for radiation dosimetry today. Despite their successful applications, the R\&D of conventional and new phosphor materials is still actively performed in a wide range of research fields from basic science to application. Although these phenomena are considered to be different, they share common physical processes such as the generation, trapping, and transfer of electronic charges. In order to have a deeper look into these phenomena, it is important to comprehensively characterize those of a single material. For this, we have constructed a prototype TSL/OSL/RPL automated and integrated measurement system (TORAIMS), and then the system performance has been studied. The system offers automated and integrated measurement of several different TSL, OSL, and RPL properties, and it is also designed to cover many different phosphor materials having a wide variety of spectroscopic properties.

\section{Introduction}

In radiation measurements, ${ }^{(1)}$ phosphors are often used as a sensing medium to convert radiation to light and/or to store radiation energy. The luminescence phenomena used in radiation measurements may vary depending on the material and application, and they are often referred to as scintillation, ${ }^{(2)}$ thermally stimulated luminescence (TSL), ${ }^{(3)}$ optically stimulated luminescence (OSL), ${ }^{(4-6)}$ and radio-photoluminescence (RPL). ${ }^{7-10)}$ Scintillation is a flash of light produced when a material interacts with ionizing radiation, so it converts radiation to light immediately. In general, the phenomenon is well understood as a recombination of radiationinduced charges at a luminescence center. In TSL and OSL, absorbed radiation energy is stored in the form of trapping charges generated by radiation. These charges are stimulated by heat or light to be freed again and then recombine at a luminescence center to emit light. The phenomena induced by heat and light stimulation are referred to as TSL and OSL, respectively. RPL is a ${ }^{*}$ Corresponding author: e-mail: go.okada@neptune.kanazawa-it.ac.jp https://doi.org/10.18494/SAM.2021.3327 
phenomenon whereby a luminescence center is generated by ionizing radiation. The specific mechanism depends on the material, while it is often interpreted to be due to charge generation and trapping at a localized center (e.g., defect or impurity ion) to act as a luminescence center.

Despite the fact that the mechanisms of the above luminescence phenomena are different, they are commonly interpreted as the generation of electronic charge by ionizing radiation, charge trapping, and recombination at a luminescence center. Earlier studies have pointed out that scintillation and storage luminescence (TSL and OSL) are inversely correlated in some phosphor materials, and TSL and OSL phenomena often share common trapping centers. ${ }^{(11-13)}$ For these reasons, for a detailed understanding of the luminescence mechanism, it is important to comprehensively study these luminescence properties. Conventionally, these properties are measured individually by separate systems. However, this approach often causes contradictions due to, for example, inconsistencies of (1) the spectral response of the photodetector, (2) the measurement orientation, (3) the sample position, etc., between different systems, as well as inconsistencies of (4) the time between irradiation and readout and (5) the irradiation geometry as it is necessary to move the sample.

In this study, in order to comprehensively characterize radiation-induced luminescence properties, we have developed an integrated and automated prototype system measuring TSL, OSL, and RPL properties, as well as scintillation. The system is named the TSL/OSL/RPL automated and integrated measurement system (TORAIMS). Note that there is a similar commercial system, the Risø TL/OSL reader, which offers automated and comprehensive measurements of TL and OSL properties. ${ }^{(14,15)}$ In addition to the features of the Risø reader, the TORAIMS offers measurements of, for example, RPL properties and scintillation properties, and some complex measurement techniques.

\section{Materials and Methods}

The TORAIMS, which offers comprehensive and automated measurement of TSL, OSL, and RPL properties, was developed in this study. Figure 1 illustrates the configuration of the TORAIMS. An X-ray generator (XRB80N, Spellman) equipped with an X-ray tube (W anode, Be window) is used as the radiation source, and the irradiation is performed downwards inside a shielded cabinet (custom-made, ANSeeN). A sample is firmly placed on an AlN ceramic heater (WALN-3H, Sakaguchi) together with an Al sample holder. The sample holder was tailormade by making a groove in a 1-mm-thick Al plate by computer numerically controlled machine processing. The thickness of the holder underneath the sample is approximately $100 \mu \mathrm{m}$, which is thin enough to ensure high thermal conduction, and the thermal gradient is negligibly small compared with that of a phosphor sample and many insulator compounds. The temperature of the heater is controlled by a modified controller unit (SCR-SHQ-A, Sakaguchi), in which the temperature controller was replaced by one with a serial port (SDC45A(C45A2A2CCR03D0), azbil). Underneath the heater, a cooling fan is located to help the ceramic heater cool to room temperature (RT) quickly. The assembly of the sample holder (including the ceramic heater) is set on a motorized vertical translation stage so that the distance from the X-ray generator during irradiation (i.e., irradiation dose rate) can be arbitrarily set. As the light source, a xenon arc lamp 


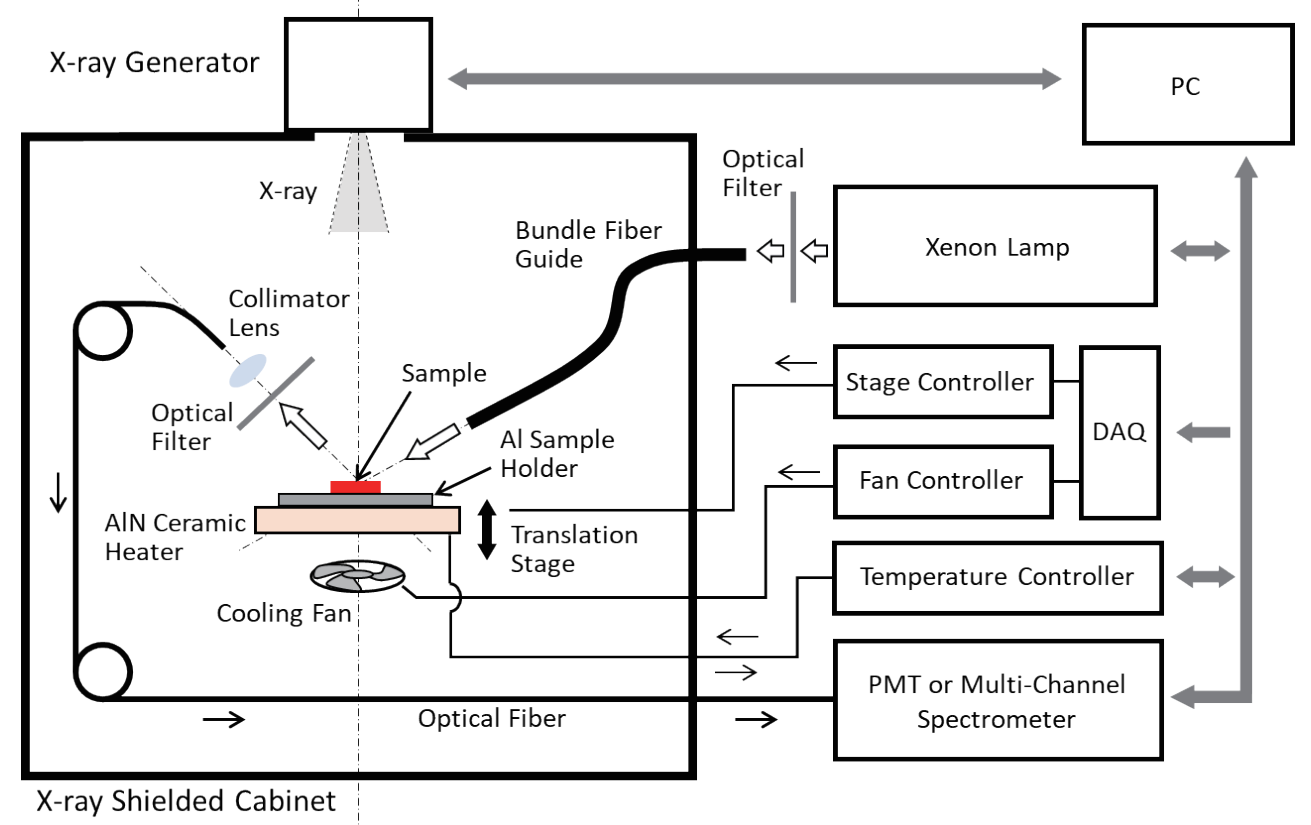

Fig. 1. (Color online) System configuration of the prototype TORAIMS (not to scale).

unit (LAX-C100, Asahi Spectra) is used. An optical band-pass filter is used to select the spectrum of light emitted from the lamp, which is then guided to the sample through a fiber bundle light guide. The emission light from the sample is collected by a collimator lens and guided to a photodetector through an optical fiber. There are two options for the photodetector: a photomultiplier tube (PMT; H11890-01, Hamamatsu) or a multichannel spectrometer (MCS; QE Pro, Ocean Optics). The former is used to detect a light signal with high sensitivity, while the latter is used to measure the spectrum. The optical filter placed in front of the collimator lens is used to select a specific spectral range of detection and/or to cut the light from the lamp. The X-ray generator, xenon lamp, translation stage, temperature, fan, PMT, and MCS are controlled by a computer via serial communication. Figure 2 illustrates the appearance of the system and a controller program. The size of the shielded cabinet is $510(\mathrm{~W}) \times 525(\mathrm{H}) \times 550(\mathrm{D}) \mathrm{mm}^{3}$. The entire system can fit on a table of size $1200 \times 750 \mathrm{~mm}^{2}$. The controller program in the figure is for measuring the TSL glow curve as a function of irradiation dose and is written in LabVIEW code. The table in the bottom left indicates the measurement sequence used to define the X-ray tube voltage and current as well as the irradiation time for each glow curve measurement. Controller programs for other measurements have a similar layout.

The important specifications of the TORAIMS are summarized in Table 1. The voltage applied to the X-ray tube can be set from 20 to $80 \mathrm{kV}$ to control the radiation energy while the tube current (from 0.12 to $1.2 \mathrm{~mA}$ ) and distance from the generator (from $\sim 0$ to $250 \mathrm{~mm}$ ) can be changed to control the dose rate. The temperature controller allows steady temperature control as well as ramp heating at different rates $\left(0.000028-1.6^{\circ} \mathrm{C} / \mathrm{s}\right)$. In general, the TSL glow curve is measured at a rate of $1.0^{\circ} \mathrm{C} / \mathrm{s}$; however, it is also important to measure at a lower heating rate in 


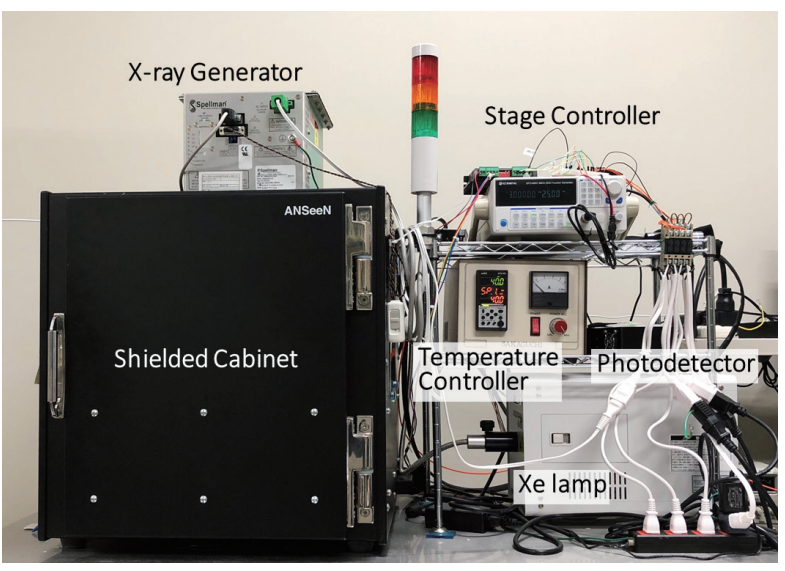

(a)

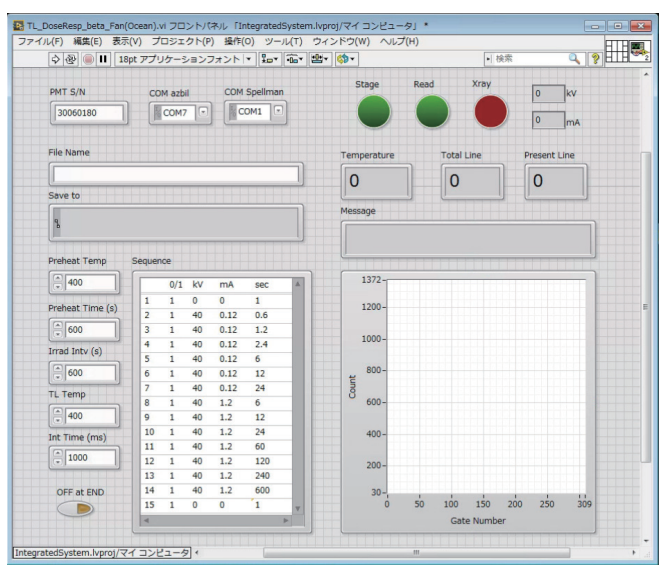

(b)

Fig. 2. (Color online) (a) Appearance of the prototype TORAIMS and (b) a controller program for TSL dose response measurement as an example.

Table 1

Important specifications of the TORAIMS.

\begin{tabular}{ll}
\hline $\begin{array}{l}\text { Radiation Source } \\
\text { Type of radiation source }\end{array}$ & X-ray tube (W anode, Be window) \\
Tube voltage & $20-80 \mathrm{kV}$ \\
Dose rate & $0.02-2.0 \mathrm{~Gy} / \mathrm{min}$ \\
\hline Light Source & \\
Type of light source & Xenon arc lamp \\
Range of wavelength & $240-1000 \mathrm{~nm}$ \\
Shutter type & Mechanical shutter \\
Shutter speed & $\sim 43 \mathrm{~ms}$ (both open and closed) \\
\hline Photodetector & \\
Spectral sensitivity range & $230-870 \mathrm{~nm}(\mathrm{PMT}) / 200-950 \mathrm{~nm}(\mathrm{MCS})$ \\
Gate time & $1-10000 \mathrm{~ms}(\mathrm{PMT}) / 8-3600000 \mathrm{~ms}(\mathrm{MCS})$ \\
Resolution (MCS) & $1.2 \mathrm{~nm}$ \\
\hline Heater & \\
Temperature range & $\mathrm{RT}-500{ }^{\circ} \mathrm{C}(\mathrm{typ}),. 600{ }^{\circ} \mathrm{C}(\mathrm{max})$. \\
Heating rate & $0.000028-1.6^{\circ} \mathrm{C} / \mathrm{s}$ \\
Sensor & $\mathrm{K}$ thermocouple \\
Sampling period & $25 \mathrm{~ms}$ \\
\hline
\end{tabular}

order to minimize the temperature gradient between the bottom and top surfaces of the sample. ${ }^{(16)}$ The temperature range of control is from RT to typically $500{ }^{\circ} \mathrm{C}\left(600{ }^{\circ} \mathrm{C}\right.$ at maximum). Figure 3(a) demonstrates a typical temperature profile during TSL measurement. In the present case, the heating rate is set to $1.0^{\circ} \mathrm{C} / \mathrm{s}$, and the temperature is ramped up from RT to $500{ }^{\circ} \mathrm{C}$. The control error of the temperature is less than $0.1{ }^{\circ} \mathrm{C}$. After the heater reaches the target temperature, it starts to cool at a rate of typically $2.0^{\circ} \mathrm{C} / \mathrm{s}$. During the cooling process, the fan is activated below $240{ }^{\circ} \mathrm{C}$ to force to lower the temperature until it reaches the original temperature. The entire measurement cycle takes about 10-15 min, depending on the 


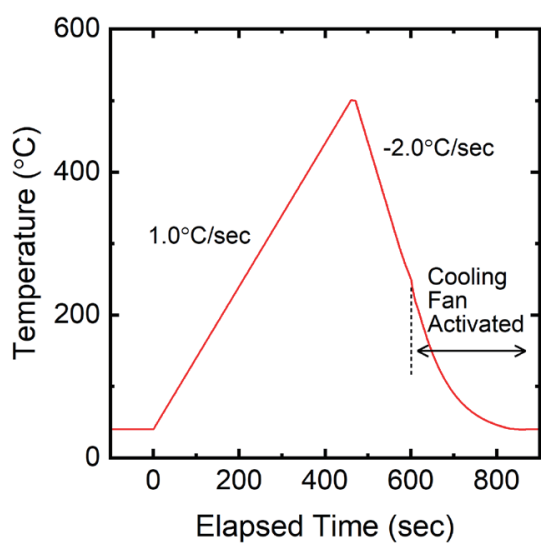

(a)

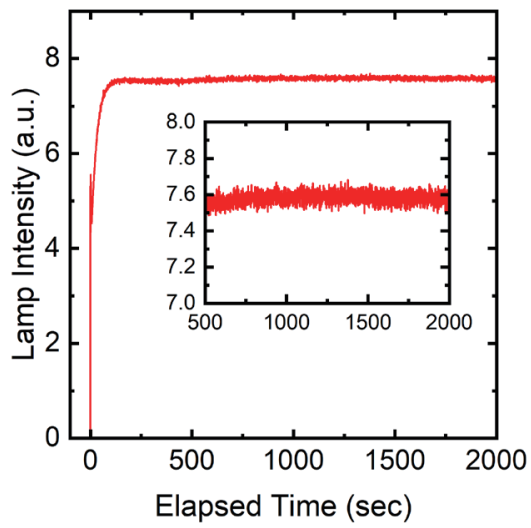

(b)

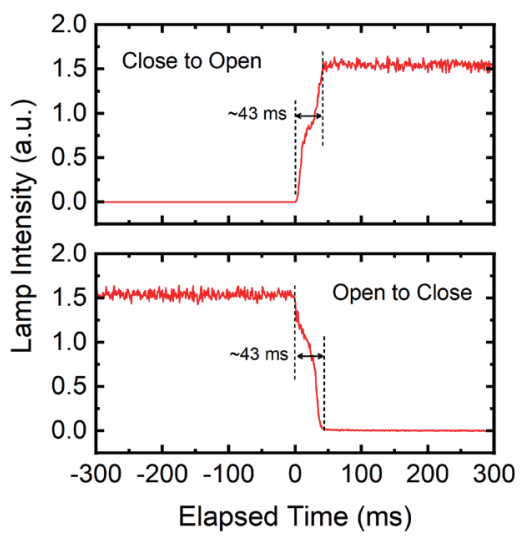

(c)

Fig. 3. (Color online) Demonstrations of (a) typical temperature profile during a cycle of TSL measurement, (b) stability of xenon lamp intensity, and (c) response of mechanical shutter.

measurement temperature range. The xenon arc light source covers the spectral range from 240 to $1000 \mathrm{~nm}$. It is equipped with a mechanical shutter and neutral density filter wheel to adjust the intensity from 5 to $100 \%$ at $1 \%$ intervals. Figure 3(b) illustrates the long-term stability of light intensity as a function of elapsed time after the lamp is powered. As demonstrated, it takes about $10 \mathrm{~min}$ for the light intensity to stabilize. The signal fluctuation is less than $3 \%$ as demonstrated in the inset. Figure 3(c) demonstrates the response of the mechanical shutter, which requires about $43 \mathrm{~ms}$ to switch from closed to open and vice versa. As the photodetector, the PMT covers the spectral range from 230 to $870 \mathrm{~nm}$ while the MCS covers from 200 to $950 \mathrm{~nm}$. The resolution of the spectrometer is approximately $1.2 \mathrm{~nm}$.

Table 2 summarizes possible measurement techniques that can be performed by using the TORAIMS. Commonly for TSL, OSL, and RPL, typical dosimetric properties such as dose response, stability, energy dependence, and reproducibility, as well as the emission spectrum can be measured. It should be noted that the system is not designed to characterize the sensitivity (or minimum detection limit) of a material itself but to provide a linear response to the dose. The 
Table 2

Possible measurement techniques using the TORAIMS.

\begin{tabular}{ll}
\hline Technique & Description \\
\hline TSL/OSL/RPL & Response vs irradiation dose \\
$\begin{array}{l}\text { Dose response } \\
\text { Stability }\end{array}$ & Response vs time after irradiation \\
Energy dependence & Response vs X-ray energy \\
Emission spectrum & Response vs measurement cycle \\
\hline TSL & Response vs emission photon wavelength \\
Glow curve & TSL vs temperature \\
Variable heating rate & Glow curve vs heating rate \\
Preheating technique & Glow curve vs treatment temperature \\
Tstop-Tmax & Max. TSL peak temperature vs preheating temperature \\
Isothermal decay curve & TSL vs time during and after irradiation vs temperature \\
Photobleaching & Glow curve after light exposure \\
OSL & \\
Decay curve & OSL vs time during stimulation \\
Temperature dependence & OSL vs temperature during readout/irradiation \\
Heat-treatment effect & OSL vs treatment temperature before/after irradiation \\
\hline RPL & PL (RPL) vs temperature during readout/irradiation \\
Temperature dependence & PL (RPL) vs treatment temperature before/after irradiation \\
Heat-treatment effect & PL (RPL) vs readout time/cycle \\
Photobleaching & \\
Others & Emission intensity during irradiation vs photon wavelength \\
Scintillation spectrum & PL vs temperature \\
Afterglow & Emission intensity vs time after irradiation with X-rays/light \\
\hline
\end{tabular}

reason is because the minimum detection limit strongly depends on the reader system, which should be designed for the individual material. To obtain an idea of sensitivity, it is more appropriate to compare the response with that of, for example, a commercial detector phosphor. For characterizing TSL properties, the glow curve, variable heating rate technique, ${ }^{(17,18)}$ preheating (partial cleaning) technique, ${ }^{(19)}$ isothermal decay curve, ${ }^{(20)}$ as well as the photobleaching effect can be performed. The TSL glow curve measurement, preheating technique, isothermal decay curve, and the photobleaching effect were demonstrated previously. ${ }^{(12,21,22)}$ In particular, for characterizing OSL properties, a luminescence decay curve during constant wave $(\mathrm{CW})$ stimulation, the heat-treatment effect, and the temperature dependence can be measured. These measurements were previously performed when characterizing OSL properties of $\mathrm{NaCl}: \mathrm{Eu}^{(12)}$ by using the TORAIMS. RPL involves the generation of a luminescence center by ionizing radiation, so the RPL properties are typically characterized by photoluminescence (PL) measurement. Using the present system, the temperature dependence, heat-treatment effect, and photobleaching effect ${ }^{(23)}$ can be measured, which were previously demonstrated on several different RPL materials. ${ }^{(24-26)}$ In particular, characterization of the temperature dependence is very important for RPL materials as it often shows so-called "build-up" of the signal, which is dependent on the post-irradiation temperature of the material. 


\section{Results and Discussion}

The TORAIMS offers automated measurements of TSL, OSL, and RPL properties; thus, it is a powerful tool, especially for R\&D of dosimetric applications, which requires not only the basic characterization of material properties but also many time-consuming and repetitive measurements such as dose response, stability, reproducibility, etc. This section demonstrates the performance of the TORAIMS for measurements of TSL, OSL, and RPL properties. LiF:Mg,Ti (TLD-100, Thermo Fisher), BeO (Thermalox995, Materion), and Ag-doped phosphate glass (AGC Techno Glass) were used for demonstrations of the TSL, OSL, and RPL measurements, respectively.

Figure 4 illustrates some example measurement results of TSL properties of LiF:Mg,Ti performed by using the TORAIMS. Figure 4(a) shows the glow curve as a function of irradiation dose, and the inset shows the dose response curve. This feature can be measured with an X-ray tube, ceramic heater, temperature controller, and PMT. The minimum detection limit is $1.0 \mathrm{mGy}$ of X-rays $(40 \mathrm{kV})$ when LiF:Mg,Ti is used. Figure 4(b) shows the TSL glow curve as a function

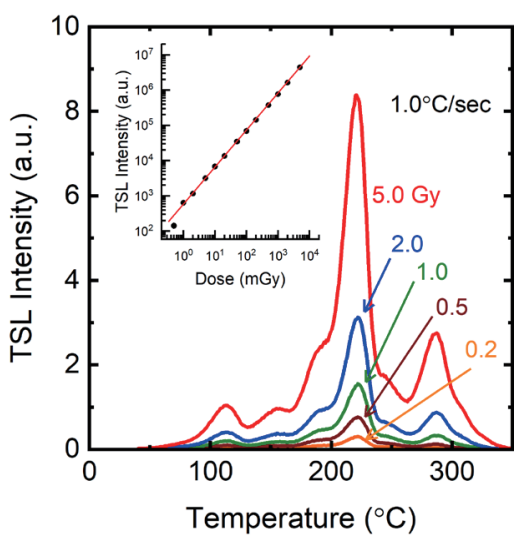

(a)

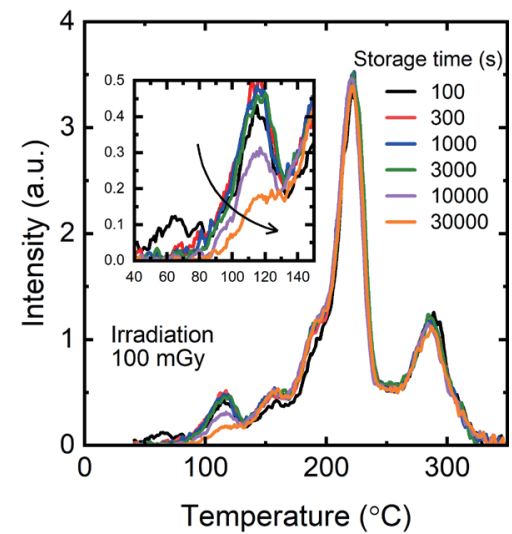

(c)

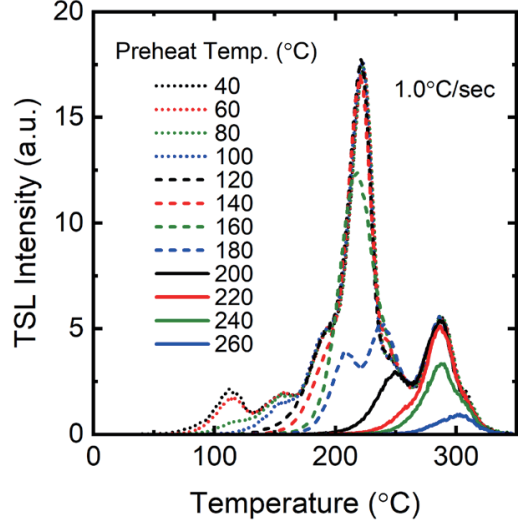

(b)

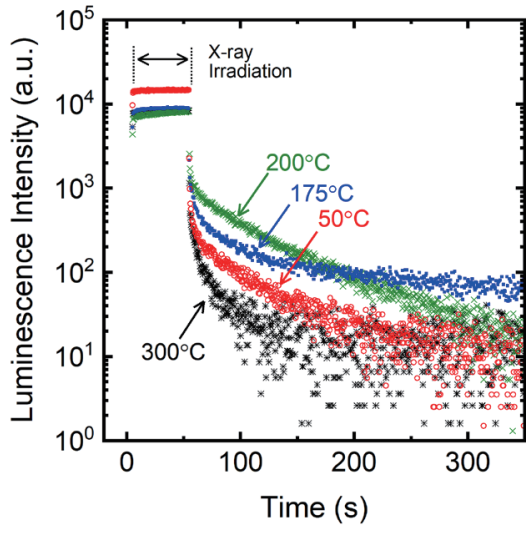

(d)

Fig. 4. (Color online) Example measurement results of TSL properties of LiF:Mg,Ti (TLD-100, Thermo Fisher). (a) TSL glow curve and dose response, (b) TSL glow curve as a function of preheat temperature, (c) TSL glow curve as a function of storage time, and (d) isothermal decay curve as a function of sample temperature. 
of preheat temperature, demonstrating that the TSL signal is partially removed by preheating, and the removed portion depends on the temperature. It is a common technique to obtain activation energies of constituting glow peaks by applying an initial rise technique to each glow curve. Figure 4(c) shows the TSL glow curve as a function of post-irradiation storage time until readout. The irradiation dose was $100 \mathrm{mGy}$ and the sample was kept at $40{ }^{\circ} \mathrm{C}$. This clearly demonstrates that, on one hand, the TSL signal at lower temperatures decreases with storage time, which is because the low-temperature signal can be stimulated by the storage temperature ( $40{ }^{\circ} \mathrm{C}$ in the present case). On the other hand, the high-temperature dosimetric peak remains consistent regardless of the storage time, which is why the high-temperature TSL signal is used for reliable dose measurement. Figure 4(d) shows isothermal TSL decay features. During the measurement, the sample is controlled at a certain constant temperature, and then the luminescence signal is recorded during and after X-ray irradiation. Typically, the decay feature can be decomposed into a sum of several exponential decay functions where each exponential function represents a trapping level stimulable at the temperature of the sample.

Figure 5 demonstrates some OSL measurement results of $\mathrm{BeO}$ performed by using the TORAIMS. Figure 5(a) shows a typical OSL decay curve as a function of irradiation dose.

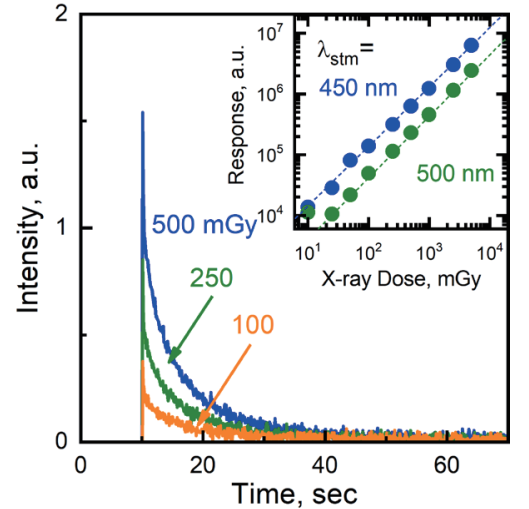

(a)



(b)



(c)

Fig. 5. (Color online) Example measurement results of OSL properties of $\mathrm{BeO}$ (Thermalox995, Materion). (a) OSL decay curves and dose response as a function of stimulation wavelength, (b) OSL decay curves as a function of stimulation wavelength, and (c) OSL response as a function of number of measurement cycles and treatment temperature prior to irradiation. 
During the measurement, the luminescence intensity was recorded while stimulating the sample with light $(450 \mathrm{~nm})$. Also, the dose response curve is illustrated in the inset. With the TORAIMS, it is possible to detect the OSL signal of $\mathrm{BeO}$ with a dose as low as $10 \mathrm{mGy}$ when irradiated with $\mathrm{X}$-rays $(40 \mathrm{kV}$ ) (a PMT is used as a photodetector). Figure 5(b) compares OSL decay curves under stimulation with light of different wavelengths $(450,500$, and $550 \mathrm{~nm})$. The stimulation wavelength is selectable by a band-pass filter used with a xenon lamp. It is clearly indicated that the OSL signal strongly depends on the stimulation wavelength. In the present case, $450 \mathrm{~nm}$ is the optimal wavelength, which is consistent with the literature. ${ }^{(27)}$ Figure 5(c) shows the OSL response signal of the measurement sample without thermal treatment and with thermal treatment at different temperatures prior to irradiation. The OSL signal gradually decreases with increasing number of measurement cycles despite the same radiation dose given to the sample. However, when the sample is treated at a high temperature before irradiation, the signal recovers (the treatment above $600{ }^{\circ} \mathrm{C}$ was performed by using a separate electric furnace). Note that the OSL signal becomes about three times larger than that without heat treatment when treated at 200 and $250^{\circ} \mathrm{C}$. The origin of this phenomenon is under investigation, but the same phenomenon was reported earlier in the literature. ${ }^{(28,29)}$

Figure 6 demonstrates some RPL measurement results performed by using the prototype TORAIMS. Figure 6(a) shows the PL spectrum of Ag-doped phosphate glass (as-irradiated and heat-treated at $140{ }^{\circ} \mathrm{C}$ for $10 \mathrm{~min}$ ) as a function of irradiation dose. It is clearly shown that the PL signal increases with the irradiation dose, indicating that the number of luminescence centers ( $\mathrm{Ag}^{2+}$ ions in the present case) increases with the irradiation dose. It also indicates that the signal strongly increases with heat treatment. ${ }^{(30-32)}$ This phenomenon is called build-up. The inset indicates the dose response functions with and without heat treatment prior to measurement. The TORAIMS enables the detection of signals with intensity as low as that when Ag-doped phosphate glass is irradiated with X-rays of a few mGy order (here, an MCS is used as a detector). The thermally assisted build-up of an RPL signal can be studied in more detail by using the TORAIMS. Figure 6(b) demonstrates PL spectra of Ag-doped phosphate glass that has been X-ray irradiated $(1.0 \mathrm{~Gy})$ and then heat-treated at different temperatures for $10 \mathrm{~min}$. The inset shows the PL intensity as a function of treatment temperature, which clearly indicates that the PL intensity is the largest after heat treatment at $140{ }^{\circ} \mathrm{C}$ but sharply decreases for heat treatment at higher temperatures. At and above $300^{\circ} \mathrm{C}$, the PL signal becomes negligibly small. Figure 6(c) shows the transition of PL intensity during the build-up as a function of treatment temperature. The slope of the build-up strongly depends on the treatment temperature. In particular, the build-up phenomenon seems to almost completely end within 10 min for heat treatment at $100{ }^{\circ} \mathrm{C}$. A previous study demonstrated the relationship between TSL and the thermally assisted build-up of the RPL signal in undoped $\mathrm{Mg}_{2} \mathrm{SiO}_{4}$ crystal. ${ }^{(33)}$ The TORAIMS allows us to reveal the correlation between TSL and RPL easily and accurately.

The TORAIMS offers not only measurements of storage luminescence properties but also measurements of X-ray-induced scintillation properties. Figure 7 demonstrates scintillation spectra of a CsI:Tl ${ }^{+}$scintillator ${ }^{(34)}$ and $\mathrm{BaFBr}: \mathrm{Eu}^{2+}$ imaging plate. These spectra were measured at RT but it is also possible to measure spectra at elevated temperatures as for the other properties. 


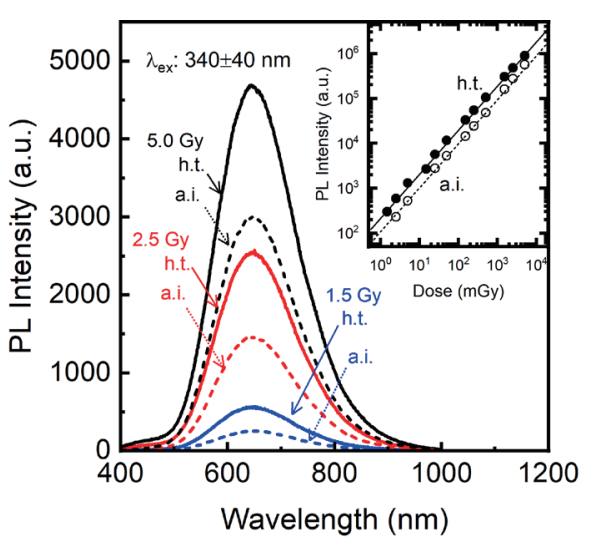

(a)

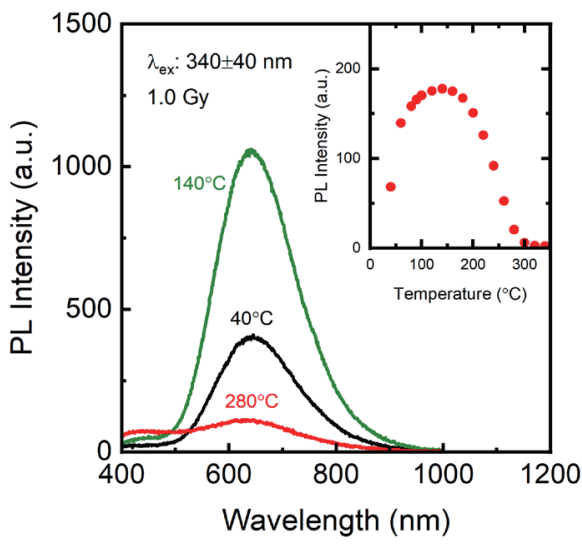

(b)

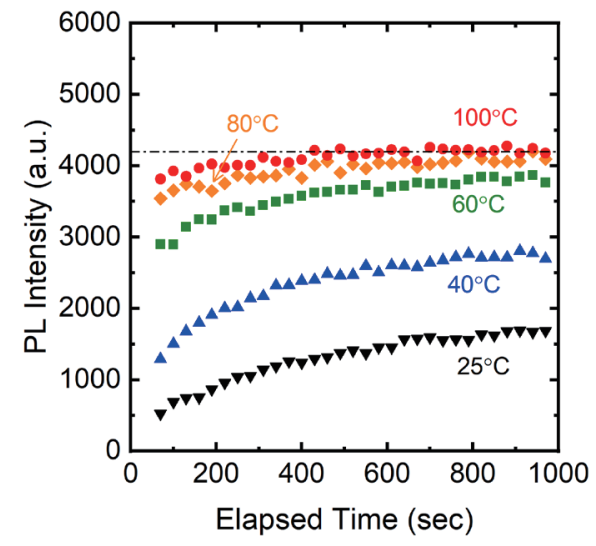

(c)

Fig. 6. (Color online) Example measurement results of RPL properties of Ag-doped phosphate glass (AGC Techno Glass). (a) PL (RPL) spectrum (as-irradiated, a.i.; heat-treated, h.t.) as a function of dose and dose response curves, (b) PL (RPL) spectrum and integrated intensity as a function of treatment temperature after irradiation and before measurement, and (c) PL intensity as a function of elapsed time after irradiation and storage temperature.

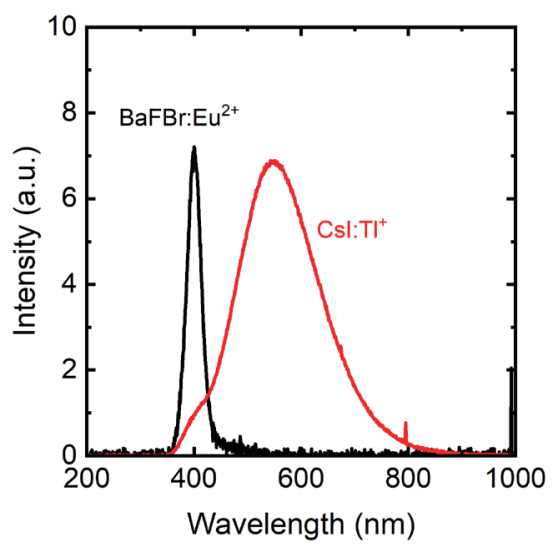

Fig. 7. (Color online) X-ray-induced scintillation spectra of CsI:Tl ${ }^{+}$scintillator (Saint-Gobain) and $\mathrm{BaFBr}: \mathrm{Eu}^{2+}$ imaging plate (Fujifilm). 


\section{Conclusions}

In this study, the TORAIMS, which allows comprehensive and automated measurements of TSL, OSL, and RPL properties, has been developed. The main components of the system are an X-ray tube, ceramic heater, xenon lamp, MCS, and PMT, and they can be controlled by a computer program in a predefined sequence. The measurement performances of the TORAIMS were demonstrated by using conventional and representative phosphor materials: LiF:Mg,Ti for TSL, BeO for OSL, and Ag-doped phosphate glass for RPL. In addition, the system offers the measurement of X-ray-induced scintillation spectra. These comprehensive measurements of luminescence properties will provide a deep understanding of the phenomena involved in radiation-induced luminescence.

\section{Acknowledgments}

We thank Mr. Y. Koguchi of Chiyoda Technol Corporation for providing Ag-doped phosphate glass and $\mathrm{BeO}$ ceramic samples to test the performance of the TORAIMS. This work was financially supported by a Grant-in-Aid for Young Scientists (B) (17K14911), a Grant-in-Aid for Early-Career Scientists (20K15207), and a Grant-in-Aid for Challenging Research (Exploratory) (19K22158) from the Ministry of Education, Culture, Sports, Science and Technology of the Japanese government (MEXT), Japan. The Cooperative Research Project of Research Institute of Electronics, Shizuoka University, and Nakatani Foundation are also acknowledged.

\section{References}

1 G. F. Knoll: Radiation Detection and Measurement (Wiley, 2010) 4th ed.

2 T. Yanagida: Proc. Japan Acad. Ser. B. 94 (2018) 75-97. https://doi.org/10.2183/pjab.94.007

3 S. W. S. McKeever: Thermoluminescence of Solids (Cambridge University Press, 1985).

4 S. W. S. McKeever: Radiat. Meas. 46 (2011) 1336. https://doi.org/10.1016/j.radmeas.2011.02.016

5 H. von Seggern: Braz. J. Phys. 29 (1999) 254. https://doi.org/10.1590/S0103-97331999000200008

6 J. A. Rowlands: Phys. Med. Biol. 47 (2002) R123. https://doi.org/10.1088/0031-9155/47/23/201

7 R. Yokota and H. Imagawa: J. Phys. 23 (1966) 1038.

8 T. Yamamoto, A. Rosenfeld, T. Kron, F. D’Errico, and M. Moscovitch: AIP Conf. Proc. 1345 (2011) 217-230. https://doi.org/10.1063/1.3576169

9 T. Yamamoto, Y. Yanagida-Miyamoto, T. Iida, and H. Nanto: Radiat. Meas. 136 (2020) 106363. https://doi. org/10.1016/j.radmeas.2020.106363

10 S. Koyama, Y. Miyamoto, A. Fujiwara, H. Kobayashi, K. Ajisawa, H. Komori, Y. Takei, H. Nanto, T. Kurobori, H. Kakimoto, M. Sakakura, Y. Shimotsuma, K. Miura, K. Hirao, and T. Yamamoto: Sens. Mater. 22 (2010) 377. https://doi.org/10.18494/sam.2010.690

11 T. Yanagida, Y. Fujimoto, K. Watanabe, K. Fukuda, N. Kawaguchi, Y. Miyamoto, and H. Nanto: Radiat. Meas. 71 (2014) 162. https://doi.org/10.1016/j.radmeas.2014.03.020

12 G. Okada, S. Motoki, M. Sakamoto, E. Kusano, R. Yada, Y. Fujimoto, T. Yanagida, and H. Nanto: J. Alloys Compd. 863 (2020) 158561. https://doi.org/10.1016/j.jallcom.2020.158561

13 T. Yanagida, G. Okada, and N. Kawaguchi: J. Lumin. 207 (2019) 14. https://doi.org/10.1016/j.jlumin.2018.11.004

14 L. Bøtter-Jensen: Int. J. Radiat. Appl. Instrumentation. Part. 14 (1988) 177. https://doi.org/10.1016/13590189(88)90060-X

15 L. Bøtter-Jensen, E. Bulur, G. A. Duller, and A. Murray: Radiat. Meas. 32 (2000) 523. https://doi.org/10.1016/ $\underline{\mathrm{S} 1350-4487(00) 00039-1}$

16 K. Shinsho, K. Otsubo, Y. Koba, K. Matsumoto, and H. Ushiba: Sens. Mater. 28 (2016) 917. https://doi. org/10.18494/SAM.2016.1252

17 G. Kitis, M. Spiropulu, J. Papadopoulos, and S. Charalambous: Nucl. Instrum. Methods Phys. Res., Sect. B 73 (1993) 367. https://doi.org/10.1016/0168-583X(93)95753-R 
18 G. Kitis and J. W. N. Tuyn: J. Phys. D. Appl. Phys. 31 (1998) 2065. https://doi.org/10.1088/0022-3727/31/16/017

19 A. Vedda, D. Di Martino, M. Martini, V. V. Laguta, M. Nikl, E. Mihokova, J. Rosa, K. Nejezchleb, and K. Blazek: Phys. Status Solidi Appl. Res. 195 (2003) 5. https://doi.org/10.1002/pssa.200306447

20 S. W. S. McKeever, J. F. Rhodes, V. K. Mathur, R. Chen, M. D. Brown, and R. K. Bull: Phys. Rev. B 32 (1985) 3835. https://doi.org/10.1103/PhysRevB.32.3835

21 K. Shinozaki, G. Okada, K. Sato, R. Yu, and M. Affatigato: J. Alloys Compd. 849 (2020) 156600. https://doi. org/10.1016/j.jallcom.2020.156600

22 N. Kawano, G. Okada, H. Kimura, and T. Yanagida: Ceram. Int. 46 (2020) 26339. https://doi.org/10.1016/j. ceramint.2020.06.092

23 G. Okada, S. Vahedi, B. Morrell, C. Koughia, G. Belev, T. Wysokinski, D. Chapman, C. Varoy, A. Edgar, and S. Kasap: Opt. Mater. (Amst) 35 (2013) 1976. https://doi.org/10.1016/j.optmat.2012.10.049

24 G. Okada, Y. Koguchi, T. Yanagida, and H. Nanto: Mater. Today Commun. 24 (2020) 101013. https://doi. org/10.1016/j.mtcomm.2020.101013

25 G. Okada, N. Kawaguchi, S. Kasap, H. Nanto, and T. Yanagida: Radiat. Meas. 132 (2020) 106251. https://doi. org/10.1016/j.radmeas.2020.106251

26 G. Okada, K. Hirasawa, E. Kusano, T. Yanagida, and H. Nanto: Nucl. Instrum. Methods Phys. Res., Sect. B 466 (2020) 56. https://doi.org/10.1016/j.nimb.2020.01.020

27 E. Bulur and H. Y. Göksu: Radiat. Meas. 29 (1998) 639. https://doi.org/10.1016/S1350-4487(98)00084-5

28 E. G. Yukihara: Radiat. Meas. 121 (2019) 103. https://doi.org/10.1016/j.radmeas.2018.12.014

29 E. G. Yukihara: Radiat. Meas. 126 (2019) 106132. https://doi.org/10.1016/j.radmeas.2019.106132

30 S. W. S. Mckeever, S. Sholom, and N. Shrestha: Radiat. Meas. 123 (2019) 13. https://doi.org/10.1016/j. radmeas.2019.02.009

31 S. Vahedi, G. Okada, B. Morrell, E. Muzar, C. Koughia, A. Edgar, C. Varoy, G. Belev, T. Wysokinski, D. Chapman, and S. Kasap: J. Appl. Phys. 112 (2012) 073108. https://doi.org/10.1063/1.4754564

32 H. Tanaka, Y. Fujimoto, M. Koshimizu, T. Yanagida, T. Yahaba, K. Saeki, and K. Asai: Sens. Mater. 28 (2016) 863. https://doi.org/10.18494/SAM.2016.1246

33 G. Okada, T. Kojima, J. Ushizawa, N. Kawaguchi, and T. Yanagida: Curr. Appl. Phys. 17 (2017) 422. https:// doi.org/10.1016/j.cap.2017.01.004

34 K. Tabata, R. Ohtake, and T. Aoki: Sens. Mater. 32 (2020) 4037. https://doi.org/10.18494/SAM.2020.2963 